\title{
Experimental Wing Damage Affects Foraging Effort and Foraging Distance in Honeybees Apis mellifera
}

\author{
Andrew D. Higginson, ${ }^{1}$ Christopher J. Barnard, ${ }^{2}$ Adam Tofilski, ${ }^{3}$ Luis Medina, ${ }^{4}$ \\ and Francis Ratnieks ${ }^{5}$ \\ ${ }^{1}$ School of Biological Sciences, University of Bristol, Woodland Road, Bristol BS8 1UG, UK \\ ${ }^{2}$ School of Biology, University of Nottingham, University Park, Nottingham NG7 2RD, UK \\ ${ }^{3}$ Department of Pomology and Apiculture, University of Agriculture in Krakow, 29 Listopada 54, 31-425 Krakow, Poland \\ ${ }^{4}$ Facultad de Medicina Veterinaria y Zootecnia, Universidad Autónoma de Yucatán, Merida, Yucatan 97100, Mexico \\ ${ }^{5}$ Department of Biology and Environmental Science, University of Sussex, Falmer, Brighton BN1 9QG, UK
}

Correspondence should be addressed to Andrew D. Higginson, a.d.higginson@bris.ac.uk

Received 23 February 2011; Accepted 19 April 2011

Academic Editor: Felipe Andrés León Contrera

Copyright ( 2011 Andrew D. Higginson et al. This is an open access article distributed under the Creative Commons Attribution License, which permits unrestricted use, distribution, and reproduction in any medium, provided the original work is properly cited.

\begin{abstract}
Bees acquire wing damage as they age, and loss of wing area affects longevity and behaviour. This may influence colony performance via effects on worker behaviour. The effects of experimental wing damage were studied in worker honeybees in observation hives by recording survivorship, how often and for how long bees foraged, and by decoding waggle dances. Mortality rate increased with both age and wing damage. Damaged bees carried out shorter and/or less frequent foraging trips, foraged closer to the hive, and reported the profitability of flower patches to be lower than did controls. These results suggest that wing damage caused a reduction in foraging ability, and that damaged bees adjusted their foraging behaviour accordingly. Furthermore, the results suggest that wing damage affects the profitability of nectar sources. These results have implications for the colony dynamics and foraging efficiency in honeybees.
\end{abstract}

\section{Introduction}

The lifespan of worker honeybees (Apis mellifera) has been reported as ranging from two to four weeks in the summer $[1,2]$ while overwintering bees that rarely leave the hive can live for several months $[2,3]$. The foraging period, defined as the length of time from the first foraging trip to death, is between seven days and two weeks [2,4-7], is not affected by the in-hive length of life before the first foraging trip [4], and is negatively related to work rate [8], but see [9].

If mortality was always due to effects extrinsic to the bee (e.g., predation and weather), then the mortality risk per unit time over a bee's foraging career would not change. Indeed, bee foragers appear to experience a constant hazard rate, as measured by the constant probability of death per unit foraging time [6]. However, [6] and other studies (see [8]), used a simple analysis that may not be accurate because it does not allow for the reduced sample size at older ages
[10]. When the same data were analysed using a Weibull plot and a more appropriate calculation of the hazard rate that controlled for the skewed data distribution, an increasing probability of death as foragers aged was apparent [10]. Re-analysis using Weibull plots also showed that increasing mortality rates during the foraging period were also present in bumblebee studies that had previously claimed a constant mortality rate [8].

It has been suggested that the flight machinery of honeybees has a fixed lifespan [4]. In an experimental study [11], removing $10 \%$ of the wing area in bumblebees results in such bees having a reduced lifespan, but the cause of mortality in these cases was unknown. However, the rate at which the flight machinery reaches some finite limit may be increased by damage to the wings, since increasing damage increases the wingbeat frequency [12]. Increasing mortality risk does not necessarily imply that lifespan is physiologically limited by the flight mechanisms [4], as age and/or wing 
damage may affect an insect's ability to avoid or escape from predators [11]. However, an increasing mortality rate in the absence of predators and inclement weather has been observed in bumblebees (Bombus terrestris, [11]), suggesting some intrinsic limit on lifespan.

If costs increase with age, we might expect bees to change their foraging strategy as they age. However, there appears to be no effect of the age of worker honey bees on foraging distance from the hive [1]. No studies have yet been carried out on the effect of age on energy use by flying insects but some have looked at the effects of wing damage. Wing damage is widespread across all insects [13] and is highly correlated with age [14-17]. In bumblebees, wing damage does not seem to affect energy use but does affect wingbeat frequency [12], because when a wing is smaller it needs to beat faster to generate more lift, but each beat requires less energy. Furthermore, wing damage has a negative effect on lifespan [11]. We have previously [18] proposed that the foraging lifespan is limited by the number of wingbeats the wing is capable of performing. If wing damage decreases lifespan by increasing the rate at which bees reach their wingbeat limit, then damaged bees may be expected to show shorter foraging periods, attempt to spend their wing beats at a slower rate and hence forage less frequently or closer to the hive, and may travel a shorter distance to forage in order to maximise the energy gain per wingbeat spent. These responses may explain such phenomena as the reduction in foraging efficiency observed after 10 days of foraging effort [19]. Also, since damaged individuals suffer increased costs associated with foraging, they may also perceive nectar sources as less profitable [20].

The current study investigated the effects of experimental wing damage on the foraging decisions of individual worker honeybees. To look for these effects, observation hives were established within which individually numbered workers could be manipulated in terms of wing damage and monitored for variation in foraging period duration and foraging behaviour. The results show that wing damage reduces survivorship, foraging trip duration and frequency, and the distance foragers travelled from the hive. Furthermore, the results suggest that wing damage might affect the perceived profitability of nectar sources, as reported by the waggle dance.

\section{Methods}

2.1. Experimental Manipulation. Data were collected in August and September of 2003 and 2004 in the Laboratory of Apiculture and Social Insects at the University of Sheffield, South Yorkshire, U.K. Each year, two colonies of 3000-5000 workers, plus queen and brood, were studied in observation hives. Honeybees were individually marked with coloured numbered disks (E. H. Thorne Ltd., U.K.) and had their wings clipped using entomological scissors to simulate wing damage (see [17]). Most bees were treated at eclosion by taking brood comb from the hives and incubating until bees emerged. Bees were introduced back to the hive via a box attached to the top of the hive separated by gauze. They were left in the box for several hours (to allow the bees to acquire the hive scent) before the gauze was removed, enabling the newly emerged bees to enter the hive. Additional bees were marked by capturing foragers as they came out of the hive and chilling them in a domestic fridge for marking and clipping of wings. These bees were placed back outside the entrance to their own hive. In 2003, all the bees were marked when they eclosed, but in 2004, the second and third cohorts were marked when adult in order to compare the effects of the time of experimental damage.

There were four treatment groups, to which a total of 855 bees were assigned randomly. Each treatment involved a different degree of wing damage as follows: Control: undamaged control, bees were handled in the same way as other bees but their wings were not clipped; Light: light damage, 3-7\% reduction in the area of each wing, and Heavy: heavy damage, $8-17 \%$ of each wing. We also attempted to damage bees asymmetrically but did not achieve a sufficiently small range of damage and had very low sample sizes throughout, resulting in unreliable results, and so these data are not presented. The amount of wing area removed from each individual was quantified using a gridded graticule by placing the wing clipping under a microscope. The wing areas removed were then converted to percentage wing damage. The area removed per wing per individual was relatively small within groups and differed among groups $\left(F_{3,219}=30.183, P<.001\right)$. The average percentage wing area removed per wing for the Heavy group was approximately twice that for the Light group. Three cohorts of 30 individuals per treatment group were treated and marked in each hive in each year.

2.2. Behavioural Observations. Between three and five times a week, marked bees were recorded by noting each bee seen during a 15 minute scan of the hive through the glass. This provided data on survivorship, including any bees that did not forage or were otherwise not observed at the hive entrance. The entrance to each observation hive was a tunnel through the outside wall of the building and a box in which there was a small camera (Micromark MM40010) that recorded the bees as they entered and exited (Figure 1). The cameras were linked to video recorders (Matsui TVR 162S) that recorded on VHS videotape from approximately 10.00$18.00 \mathrm{~h}$ each day of observation to yield the daily time each bee spent outside the hive and the number of times it left the hive.

Waggle dances were recorded opportunistically onto DAT tape using a digital video recorder (Sony DCR TRV 16E) by an observer watching the colonies from approximately $10.00-18.00 \mathrm{~h}$ each day of observation. The cameras were focused on the dancing area, the half of the lower comb near the hive entrance and positioned to maintain the dance floor in full plane view on the screen. The camera was held on a retort stand and clamp, so was steady but movable. Upon the observation of a waggle dance by a marked individual, the camera was moved if necessary to keep the dance in the centre of the screen. The recording continued until the focal individual stopped dancing or moved off the dance floor. Vertical lines $5 \mathrm{~cm}$ apart were drawn on the outside 


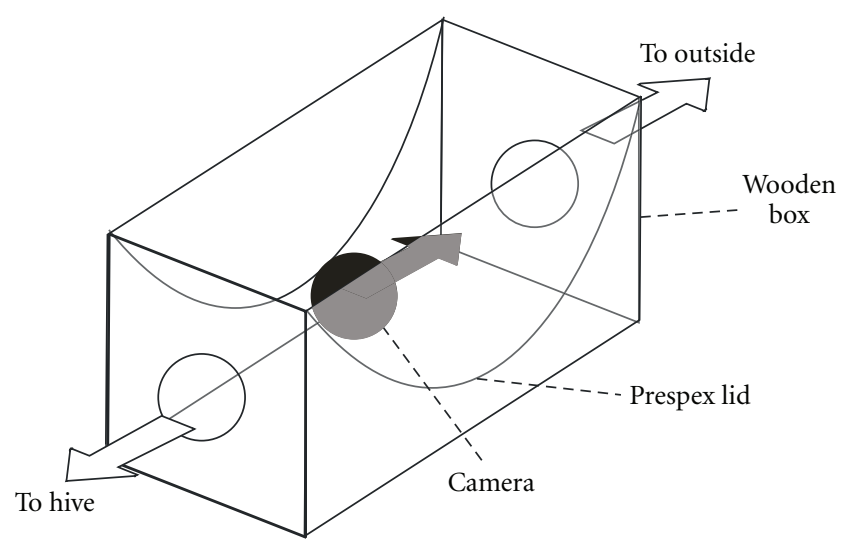

FIgURE 1: The camera box that bees must pass though to leave the hive. Bees are forced in the lower half of the box, under the curved perspex lid. This design forces all bees entering and exiting the hive to show their backs (and hence any numbered disc) to the camera positioned on top of the perspex lid.

of the observation hive in thin permanent marker to enable calibration of the angle of the dance to vertical.

All recordings were converted to Quicktime (Apple Computer, Inc., Cupertino, CA, USA) format, and the dances were cropped from the film. The dances were analysed using the Videopoint (Lenox Softworks, Inc., Lenox, MA, USA) path recording software. The program combines a spreadsheet and video. The user clicks on any part of the image to record the relevant pixels in the spreadsheet, yielding coordinates and frame number. Thus, for instance, clicking on the head of the bee at the start and the end of the waggle run of the dance would provide the duration and angle of the run. Additionally, clicks before each dance on the top and the bottom of a drawn line on the outside of the hive recorded the vertical, to control for imperfect orientation of the camera. The spreadsheet was entered into Microsoft Excel where the data were compressed, deleting all the frames where no click occurred. From the four recorded points (start of vertical line, end of vertical line, start of waggle run, end of waggle run), it was possible to calculate the angle of the waggle run on the honeycomb and its duration. Multiple waggle runs of the dance by each bee were analysed.

The waggle dance comprises a rough figure of eight pattern on the comb, with the direction of movement of the dancer tending to alternate [21]. Three main kinds of information are passed on to the waggle dance follower [20, 22]. The duration of the waggle run represents the distance between the hive and the food source, with one second corresponding to $500-1400 \mathrm{~m}$, with significant variation between subspecies and landscapes $[2,22,23]$. The angle of the waggle dance relative to the vertical represents the angle of the flight path relative to the solar azimuth. The duration and rate of performing the waggle run, and, therefore, the time between each waggle run, is related to the profitability of the food source in that the shorter the time between waggle runs the more profitable the nectar source [20]. The mean waggle run duration and direction were calculated for each waggle dance. The angle of the dance on the comb was translated by means of a program (Sun 97 provided by W. F. Towne) that calculated the position of the solar azimuth at the time of each dance. The frequency of wagglerun production was estimated as the mean length of time between waggle runs.

Data were analysed by ANOVA, with normalising transformations as necessary, to check for differences in survivorship, foraging effort, foraging distance and reported quality between the treatment groups. Where normalization was not possible, equivalent nonparametric tests were used. Hazard rate and Weibull analysis (to assess mortality over time) followed the procedure detailed in [10]. Hutchinson [10] suggested that survivorship should be plotted using the Weibull distribution to see if it differed from an exponential distribution. If mortality risk is constant, the number of deaths will be exponential, which yields a gradient of unity when the Weibull distribution is plotted. Analysis was carried out in SPSS (SPSS Inc., Chicago, IL, USA) and R (Free Software Foundation, Boston, MA, USA). Unless otherwise stated there were no differences between colonies so data were pooled for most analyses.

\section{Results}

3.1. Survivorship and Hazard Rate. The first and last occasions on which individuals with known date of eclosion were noted in the hive and at the entrance provided an estimate of lifespan. We found that Control bees lived for around 20.5 days on average, whereas experimentally damaged bees lived for around 18 days, and there was no difference between the two levels of wing area removal (Figure 2(a)). The difference between treatment groups in lifespan was statistically significant (Kruskal-Wallis test: $H 4=14.415, P=.006)$.

The probability of mortality per day (hazard rate) was initially low at around 0.02 but showed an accelerating increase as bees age such that for the oldest foragers it was greater than 0.25 (Figure 2(b)). The hazard rate actually fits an exponential distribution $\left(F_{1,26}=45.65, P<.001\right)$, showing a sharp increase in mortality at around 28 days of age. Furthermore, damaged bees experienced a $50 \%$ higher hazard rate when young, but around a $25 \%$ higher hazard rate when older. These observations are confirmed by fitting hazard rate models. The data provided a better fit to the Weibull distribution than to an exponential distribution $(F=402.6, d f=1, P<.001)$, implying that the hazard rate increased over time. Furthermore, a model fitting slopes to the three treatment groups provided a better fit than a model that assumed a single slope for all groups $(F=8.99, d f=$ $1, P=.011)$, but was not a better fit than a model that fitted a single slope to both the Light and Heavy groups together $(F=1.515, d f=1, P=.218)$, implying that their hazard rates did not differ. That is, both groups of artificially damaged bees experienced a consistently higher mortality rate than control bees, but all individuals were subject to an increasing risk of mortality as they aged.

3.2. Length of Foraging Trip. The 288 hours of video data of bees entering and exiting the hive were subsampled using the random number generator in Microsoft Visual Basic 


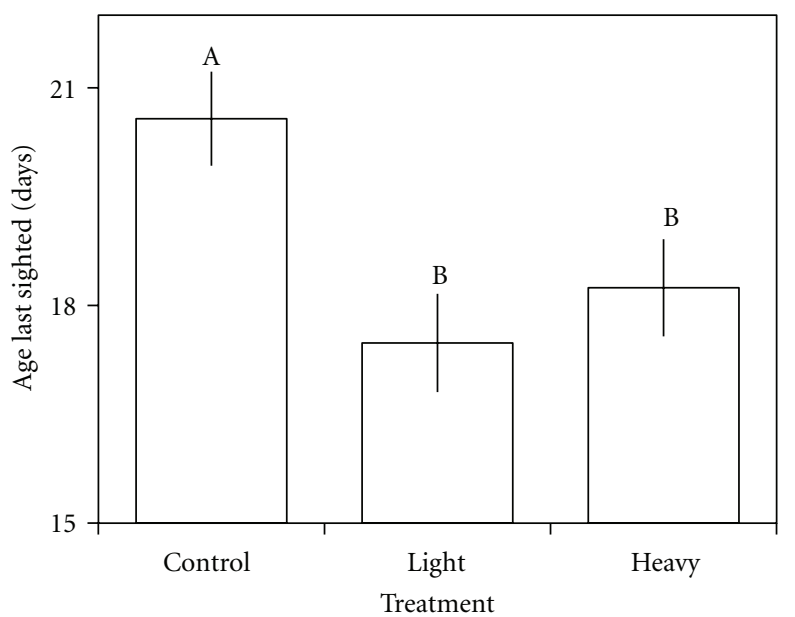

(a)

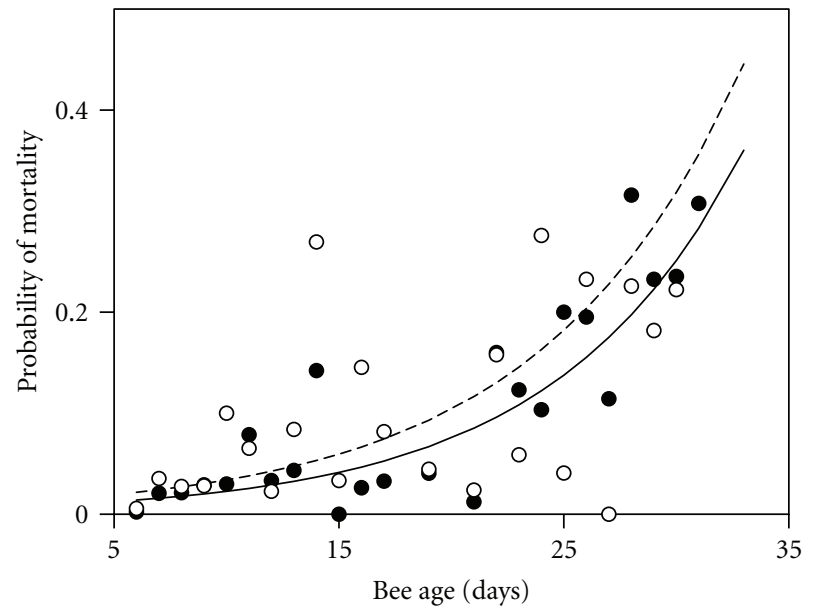

(b)

FIGURE 2: (a) Mean ( \pm 1 SE) lifespan of workers in the treatment groups. Bars that share the same letter did not significantly differ. (b) Hazard rate (probability of death per day) against bee age for Control (closed circles, solid line) and Light treatments (open circles, dashed line). The lines represent exponential fits of the data $\left(0.0068 \mathrm{e}^{0.1 t}, 0.0148 \mathrm{e}^{0.0861 t}\right.$, respectively). Only two groups are shown for the sake of clarity and the curve for the Heavy group did not differ significantly from that for the Light group.

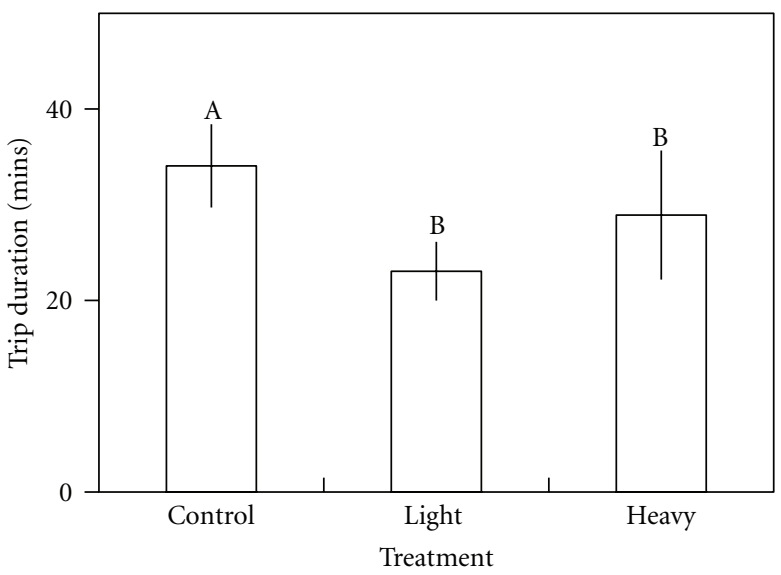

(a)

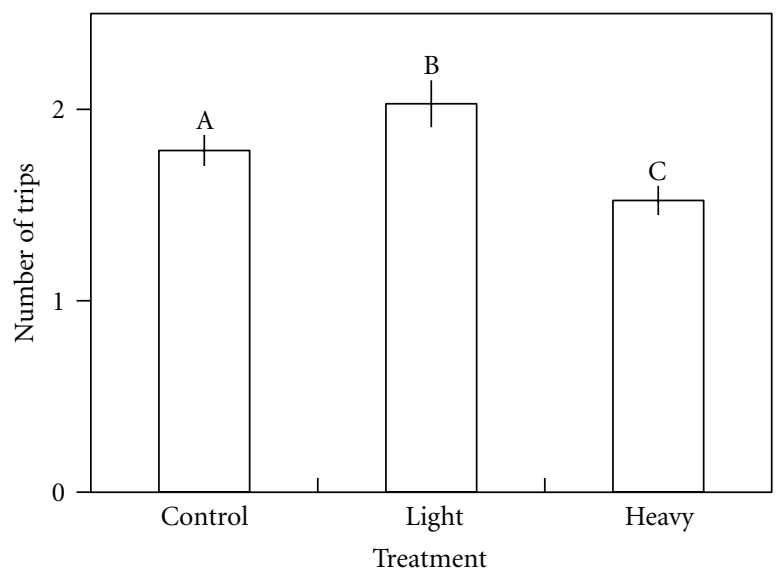

(b)

Figure 3: Mean ( \pm 1 SE) (a) duration in minutes and (b) number of foraging trips per bee for each treatment group. Bars that share the same letter did not significantly differ using post hoc tests.

(Microsoft Inc, Redmond, WA, USA). The data on marked bees entering and exiting the hive was used to calculate the duration of 172 foraging trips (up to five for any given bee). Trips were omitted from analysis (a) if they were shorter than two minutes (31 trips), since it was likely that the bee had not left the outside tunnel, and (b) if they were longer than 3 hours, since the bee was likely to have been missed when reentering the hive ( 8 trips).

ANOVA of trip duration (natural log transformed to normalise) with colony and treatment group as factors and date and time of day as covariates showed there was a difference between the two colonies in the duration of foraging trips $\left(F_{1,163}=3.98, P=.048\right)$. There was a positive effect of date $\left(F_{1,163}=25.92, P<.001\right)$, and a negative effect of time of day $\left(F_{1,163}=5.903, P=.016\right)$ on trip duration; trips became longer over the observation period, and shorter trips were carried out in the afternoon. Trips carried out before noon were more than twice as long, on average, as those carried out after $2 \mathrm{pm}$. Crucially, there was a difference between the treatment groups in trip duration $\left(F_{3,163}=3.518, P=.016\right)$. Trips carried out by Control bees were almost $50 \%$ longer, on average, than those carried out by Light bees and $17 \%$ longer than those carried out by Heavy bees (Figure 3(a)). To look for potential effects of bee age, the same analysis was carried out on the bees whose date of eclosion was known, but there was no effect of age on trip duration $\left(F_{1,123}=0.406, P=.525\right)$. The number of foraging trips recorded for each bee also differed between treatments (Kruskal-Wallis test: 18.2, $N=287, P<.001$ ): Heavy bees performed around 17\% fewer trips than Control bees and 


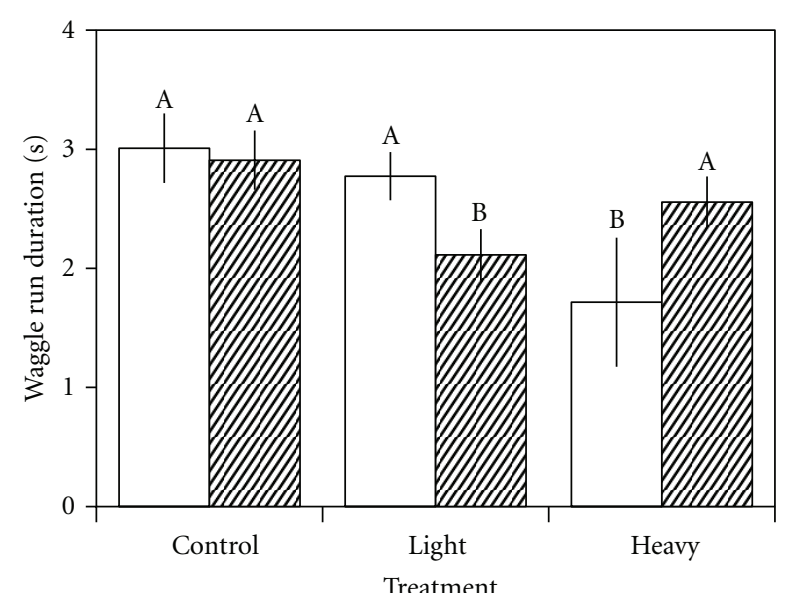

(a)

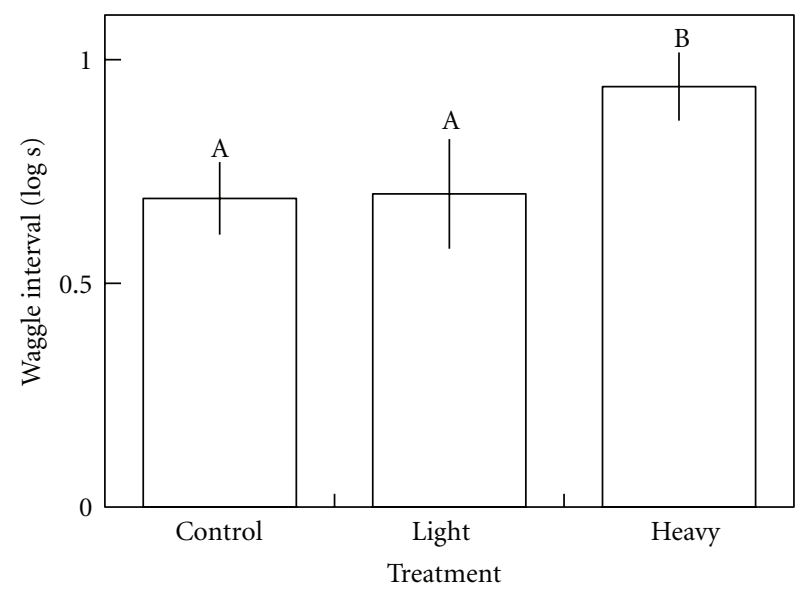

(b)

FIgURE 4: (a) Mean (+1 SE) duration of waggle run per dance for each treatment group and whether they were damaged after they started foraging (open bars) or at eclosion (hatched bars). (b) Mean ( $\pm 1 \mathrm{SE}$ ) interval between waggle runs per dance (return run) for the three treatment groups. Bars that share the same letter did not significantly differ. Heavy bees indicated lower quality nectar sources than Control and Light bees.

33\% fewer trips than Light bees, and Control bees performed 13\% fewer trips than Light bees (Figure 3(b)).

3.3. Choice and Perceived Quality of Patches. The observations provided 828 waggle runs from 164 dances by 132 individuals (up to three dances per bee). ANOVA showed that there was a difference in waggle run duration between colonies $\left(F_{3,152}=3.11, P=.047\right)$ and a difference between treatment groups $\left(F_{3,152}=7.463, P<.001\right.$, Figure $4(a)$ ): whilst waggle run duration was approximately equal for the two groups of damaged bees, it was $20 \%$ shorter, on average, than for Control bees. The same analysis was carried out on the bees whose dates of eclosion were known, with age as a covariate, but there was no significant effect of bee age on run duration $\left(F_{1,75}=2.427, P=\right.$ .123). There was also no difference in waggle run duration between bees that had been treated at eclosion and those that were treated after they started foraging $\left(F_{1,128}=\right.$ $0.061, P=.806$ ), but there was a significant interaction between this factor (time of manipulation) and treatment group $\left(F_{3,128}=3.598, P=.015\right)$, Heavy individuals that were wing clipped after they started foraging and Light individuals clipped at eclosion travelled shorter distances than other groups, whereas there was no difference in distance between the two times of manipulation for the Control bees (Figure 4(a)). Wing clipping at eclosure rather than after foraging started was associated with a $23 \%$ reduction in wagglerun duration in Light bees, but a $48 \%$ increase in Heavy bees.

ANOVA (using dances where at least three waggle runs were recorded) was performed on the mean length of time between runs (normalised by natural logarithms), with treatment group, colony and time of manipulation (eclosion or adulthood) as factors, and mean waggle-run duration as a covariate, the latter to assess whether reported profitability and distance were related. There were no differences between colonies $\left(F_{3,120}=0.0082, P=.93\right)$ nor an effect of the time of manipulation $\left(F_{1,120}=1.34, P=.24\right)$. There was, however, a positive relationship between the mean length of time between waggle runs and mean waggle run duration $\left(F_{1,120}=10.58, P=.0014\right)$; nectar sources further from the hive were reported to be less profitable. There was also a significant difference between treatment groups $\left(F_{3,120}=\right.$ $4.26, P=.041)$; Heavy damaged bees reported lower profitability of nectar sources (Figure 4(b)). Interestingly, there was an interaction between treatment and waggle run duration $\left(F_{3,120}=3.93 ; P=.05\right)$; the slope relating waggle run duration and time between waggle runs became less positive as the amount of added wing damage increased across groups. Hence, while control bees perceived more distant sources as less profitable, both groups of damaged bees perceived all sources as less profitable than controls, and even when nectar sources were at the same distances.

Fifty-three bees were observed both dancing and carrying out foraging trips, but there was no relationship between trip duration and waggle-run duration $\left(r_{53}=-0.051, P=\right.$ $.719)$ or return run duration $\left(r_{53}=-0.007, P=.958\right)$; trips to sites further away did not take longer. This might be unsurprising since bees will spend much more time foraging within a flower patch than flying to and from the patch.

\section{Discussion}

The results suggest that experimentally induced wing damage had deleterious effects on the survivorship and foraging behaviour of honeybees, and since wing damage is acquired naturally $[13,17]$, this has implications on foraging ecology. Since many other insects acquire wing damage, these effects may have analogues in an ecologically and taxonomically broad range of species.

The effects of experimental wing damage on lifespan expand on the results of other studies. Hutchinson (2000) 
plotted the hazard rate against foraging life duration for the data of [6] and showed that there was a sharp increase in the final ( 80 hour) age group of their bees. This could be because many bees do not die as a result of predation but do reach a physiological limit, and this was typically after 80 hours foraging times in those data [6]. The data in the present study were based on total lifetime so they include one or two weeks before the foraging period starts. Therefore, they are not directly comparable with the foraging period duration data of [6], but they do show a similar pattern, namely a sharp increase in hazard rate late in life, after 25 days in our study. Caution must be exercised in interpretation because the end of the curve is based on little data, as few bees are still alive, and so is imprecise [10], but the data suggest there is a finite limit on foraging performance or lifespan. As experimentally damaged bees apparently died sooner than undamaged bees, the results suggest they may have reached this limit sooner. Since damaging wings is likely to have required an increase in wingbeat frequency [12] throughout the bees' lifetime, the flight mechanisms may have worn out sooner. If damage simply reduced the ability of bees to avoid predators, the probability of predation would not change as the bees aged, so there would have been no increase in hazard risk over time. We also found that the hazard rate for experimentally damaged bees increased more rapidly than that for undamaged individuals, as previously observed by [11], (reanalysed by [8]), suggesting that damaged bees reach such a physiological limit sooner.

Similar survivorship curves have been obtained for several bumblebee (Bombus spp.) species. Estimates of mean lifespan vary between 13 and 41 days $[9,24,25]$, but all show increasing mortality rate with age. As [25] state "mortality rate was nearly constant for the first 14 days of adult life, after which it increased sharply." This suggests that there is a physiological limit on longevity in social insects, and this is true in laboratory conditions in the absence of weather or predation [8]; longevity has also been shown to be inversely related to physical activity in other insects, such as the house fly Musca domestica [26] and Drosophila [27]. A limit on flight performance would also explain the inverse relationship between work rate, or amount of flying, and longevity of bees $[4,24,28]$.

The hypothesis that there is a limit on flight performance was also supported by the other findings of the present study. There was no effect of age on the length of foraging trips or the distance travelled from the hive, in line with [1]. However, there were clear effects of experimental wing damage on trip duration and distance travelled in that trips and distances were longest for undamaged bees. The finding that damaged bees travelled shorter distances suggests that flight is more costly and, therefore, foraging trips are less profitable at greater distances. It is possible that flight speed was affected by damage, or that damaged bees took longer on foraging trips because of a reduction in foraging ability. Earlier work by us [17] has shown that damaged bees were less choosy, which may have resulted in poorer gain rates, and so damaged bees to return to the hive quicker to find alternative nectar sources. Since there is an effect of the weight being carried on wingbeat frequency and energy expenditure $[29,30]$, damaged bees may be more limited in how much they can carry.

However, since energy requirement does not increase with reduced wing area [12], these behavioural responses cannot be related to energetic efficiency or net rate. Wingbeat frequency has been shown to increase with reduction in wing area [12], so in both deciding on how far to travel, and when to return to the hive, bees may use a currency of energetic gain to per wingbeat [18]. This is in line with previous findings of times of returning to the hive that assumed a currency of energetic efficiency (net energy gain per unit energy spent), (e.g., [31]).

These findings also tie in well with our results showing that undamaged bees reported patches further from the hive as less worthwhile, which may be because patches further from the hive happened to be poorer, or because more costs are incurred in travelling to and from the patch. The interaction between treatment and distance on profitability is in line with this. Control bees report more distant patches as less profitable, but damaged bees report all patches as less profitable. If it is the case that damaged bees reported profitability from foraging trips differently from undamaged bees, it will mean that individual wing damage is likely to have colony-wide effects on foraging.

The differences between the damage treatment groups were interesting in that heavily damaged individuals carried out foraging trips of lengths intermediate between the controls and lightly damaged but carried out fewer trips. As they seemed to carry out fewer trips than lightly damaged individuals, it appeared that light damage caused shorter but more frequent trips, but heavy damage causes less frequent trips. These findings highlight the need for further experimental study in to the effects of wing damage on flight costs and load carriage. It was surprising that there were no differences between lightly damaged and heavily damaged individuals in survivorship and foraging distance. One might expect that doubling the amount of wing damage would at least increase the effects of damage. There may be a general effect of damage to the bee not due to the amount of wing removed, although our control should have controlled for handling effects. Alternatively, the effect of damage may be non linear, and the damage added here was sufficient to reach the saturation point of the behavioural response. Further work with finer differences between treatments are needed to test this possibility. Heavily damaged bees may have performed fewer foraging trips than lightly damaged bees, therefore, slowing the rate of exhaustion of the mechanisms of flight and, hence, surviving for the same length of time. A difference in work effort may explain the difference in the change in the hazard rate between the control and damaged groups, if undamaged bees flew further or carried out longer trips, and so the behaviour of damaged bees gradually over time reduced the apparent effect of damage on the hazard rate shown in Figure 2(b).

Overall, the results suggest there is strong selective pressure for honeybees to avoid accumulating wing damage, since it has marked apparent effects on the foraging efficiency of colonies, from flower choice and patch choice, to foraging time and even lifespan. As honeybees may avoid foraging 
in vegetation-dense areas to reduce their risk of sustaining wing damage [32], these effects are likely to have profound implications for the impact of the spatial distribution, density and plant architecture of food sources exploited by bees.

\section{Acknowledgments}

We are grateful to everyone in the Laboratory of Apiculture and Social Insects for stimulating discussion and technical assistance and Anaid Diaz-Palacios for statistical advice. Lars Chittka and four anonymous referees provided advice on an earlier version of this manuscript. This work was funded by a BBSRC Committee Studentship to A. D. H., and complied with the laws of the U.K. concerning the experimental use of animals. A. D. H is currently supported by E.R.C. Advanced Grant 250209 awarded to Alasdair Houston.

\section{References}

[1] N. E. Gary, P. C. Witherall, and K. Lorenzen, "Effect of age on honeybee hymenoptera, apidae foraging distance and pollen collection," Environmental Entomology, vol. 10, no. 6, pp. 950952, 1981.

[2] T. D . Seeley, Honeybee Ecology, Princeton University Press, Princeton, NJ, USA, 1985.

[3] R. E. Page Jr. and C. Y.-S. Peng, "Aging and development in social insects with emphasis on the honey bee, Apis mellifera L," Experimental Gerontology, vol. 36, no. 4-6, pp. 695-711, 2001.

[4] A. Neukirch, "Dependence of the life span of the honeybee (Apis mellifica) flight performance and energy consumption," Journal of Comparative Physiology B, vol. 146, no. 1, pp. 35-40, 1982.

[5] T. J. Wolf, P. Schmid-Hempel, C. P. Ellington, and R. D. Stevenson, "Physiological correlates of foraging efforts in honey-bees: oxygen consumption and nectar load," Functional Ecology, vol. 3, no. 4, pp. 417-424, 1989.

[6] P. K. Visscher and R. Dukas, "Survivorship of foraging honey bees," Insectes Sociaux, vol. 44, no. 1, pp. 1-5, 1997.

[7] A. Tofilski, "Senescence and learning in honeybee (Apis mellifera) workers," Acta Neurobiologiae Experimentalis, vol. 60, no. 1, pp. 35-39, 2000.

[8] D. E. Adair, Physiological Senescence, Predatory Pressure and the Foraging Behaviour of Bees, Ph.D. thesis, University of Cambridge, Cambridge, UK, May 2000.

[9] P. Schmid-Hempel and T. J. Wolf, "Foraging effort and life span of workers in a social insect," Journal of Animal Ecology, vol. 57, no. 2, pp. 509-521, 1988.

[10] T. P. Hutchinson, "Graphing the survivorship of bees," Insectes Sociaux, vol. 47, no. 3, pp. 292-296, 2000.

[11] R. V. Cartar, "Morphological senescence and longevity: an experiment relating wing wear and life span in foraging wild bumble bees," Journal of Animal Ecology, vol. 61, no. 1, pp. 225-231, 1992.

[12] A. Hedenström, C. P. Ellington, and T. J. Wolf, "Wing wear, aerodynamics and flight energetics in bumblebees (bombus terrestris): an experimental study," Functional Ecology, vol. 15, no. 4, pp. 417-422, 2001.

[13] R Dudley, The Biomechanics of Insect Flight: Form, Function and Evolution, Princeton University Press, Princeton, NJ, USA, 2000.
[14] S. S. Ragland and R. S. Sohal, "Mating behavior, physical activity and aging in the housefly, Musca domestica," Experimental Gerontology, vol. 8, no. 3, pp. 135-145, 1973.

[15] K. L. H. Leong, D. Frey, D. Hamaoka, and K. Honma, "Wing damage in overwintering populations of monarch butterfly at two California sites," Annals of the Entomological Society of America, vol. 86, no. 6, pp. 728-733, 1993.

[16] D. U. Burkhard, P. I. Ward, and W. U. Blanckenhorn, "Using age grading by wing injuries to estimate size-dependent adult survivorship in the field: a case study of the yellow dung fly Scathophaga stercoraria," Ecological Entomology, vol. 27, no. 5, pp. 514-520, 2002.

[17] A. D. Higginson and C. J. Barnard, "Accumulating wing damage affects foraging decisions in honeybees (Apis mellifera L.)," Ecological Entomology, vol. 29, no. 1, pp. 52-59, 2004.

[18] A. D. Higginson and F. Gilbert, "Paying for nectar with wingbeats: a new model of honeybee foraging," Proceedings of the Royal Society B, vol. 271, no. 1557, pp. 2595-2603, 2004.

[19] R. Dukas and P. K. Visscher, "Lifetime learning by foraging honey bees," Animal Behaviour, vol. 48, no. 5, pp. 1007-1012, 1994.

[20] T. D. Seeley, A. S. Mikheyev, and G. J. Pagano, "Dancing bees tune both duration and rate of waggle-run production in relation to nectar-source profitability," Journal of Comparative Physiology, vol. 186, no. 9, pp. 813-819, 2000.

[21] K. von Frisch and M. Lindauer, "The "language" and orientation of the honeybee," Annual Review of Entomology, vol. 1, pp. 45-48, 1956.

[22] K. von Frisch, The Dance Language and Orientation of Bees, Harvard University Press, Cambridge, Mass, USA, 1967.

[23] M. V. Srinivasan, S. W. Zhang, M. Altwein, and J. Tautz, "Honeybee navigation: nature and calibration of the "odometer"," Science, vol. 287, no. 5454, pp. 851-853, 2000.

[24] E. V. da Silva-Matos and C. A. Garófalo, "Worker life tables, survivorship, and longevity in colonies of Bombus (fervidobombus) atratus (hymenoptera: apidae)," Revista de Biologia Tropical, vol. 48, no. 2-3, pp. 657-664, 2000.

[25] F. H. Rodd, R. C. Plowright, and R. E. Owen, "Mortality rates of adult bumble bee workers (hymenoptera: apidae)," Canadian Journal of Zoology, vol. 58, pp. 1718-1721, 1980.

[26] R. S. Sohal and P. B. Buchan, "Relationship between physical activity and life span in the adult housefly, Musca domestica," Experimental Gerontology, vol. 16, no. 2, pp. 157-162, 1981.

[27] L. Partridge and M. Farquhar, "Sexual activity reduces lifespan of male fruitflies," Nature, vol. 294, no. 5841, pp. 580-582, 1981.

[28] M. Rockstein, "Longevity in the adult worker honeybee," Annals of the Entomological Society of America, vol. 43, pp. 152$154,1950$.

[29] C. P. Ellington, "The aerodynamics of hovering insect flight. III. lift and power requirements," Philosophical Transactions of the Royal Society B, vol. 305, no. 1122, pp. 145-181, 1984.

[30] T. J. Wolf and P. Schmid-Hempel, "Extra loads and foraging life span in honeybee workers," Journal of Animal Ecology, vol. 58, no. 3, pp. 943-954, 1989.

[31] P. Schmid-Hempel, A. Kacelnik, and A. I. Houston, "Honeybees maximize efficiency by not filling their crop," Behavioral Ecology and Sociobiology, vol. 17, no. 1, pp. 61-66, 1985.

[32] A. D. Higginson, Effects of wing damage on the behaviour of the honeybee Apis mellifera, Ph.D. thesis, University of Nottingham, Nottingham, UK, 2005. 

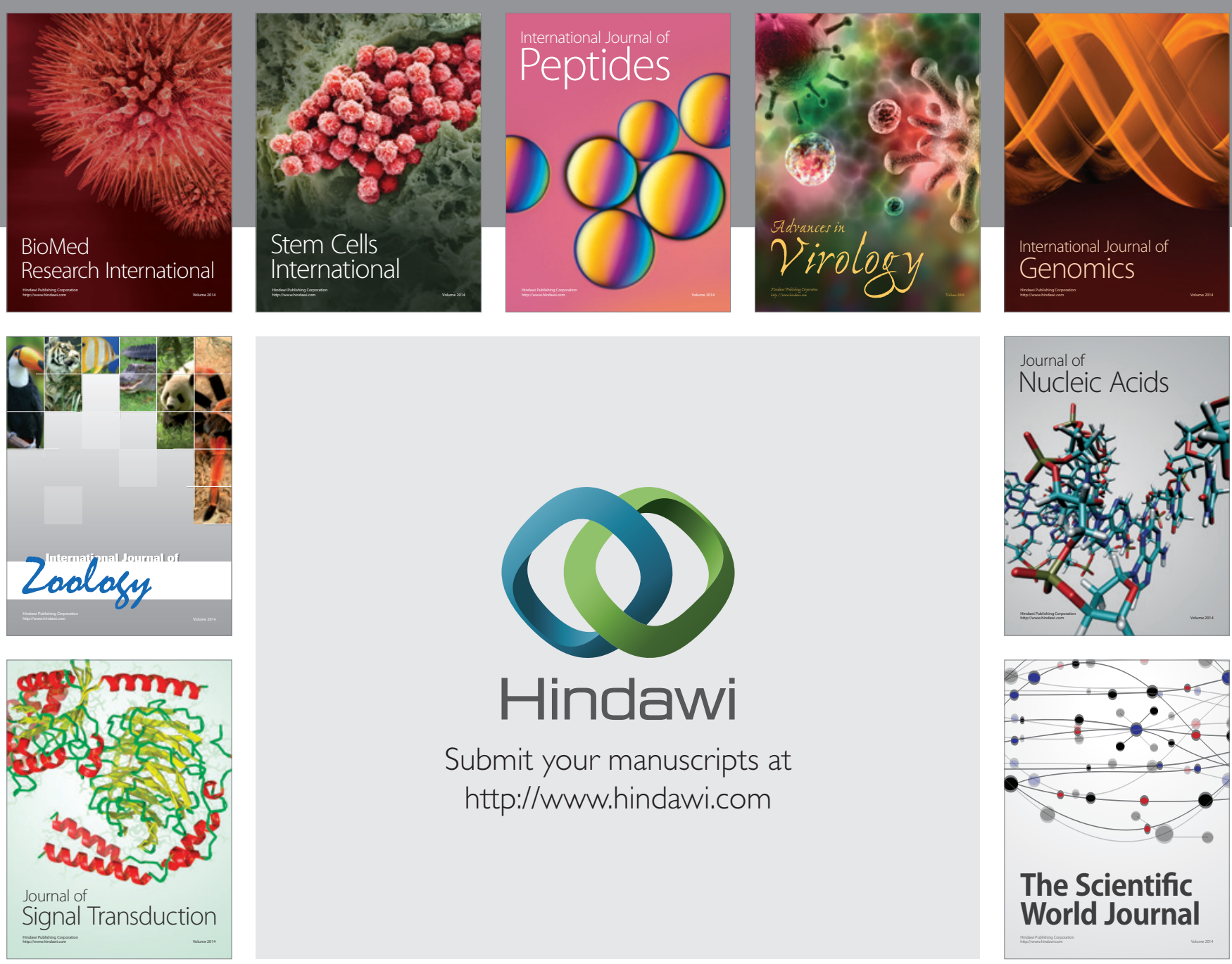

Submit your manuscripts at

http://www.hindawi.com
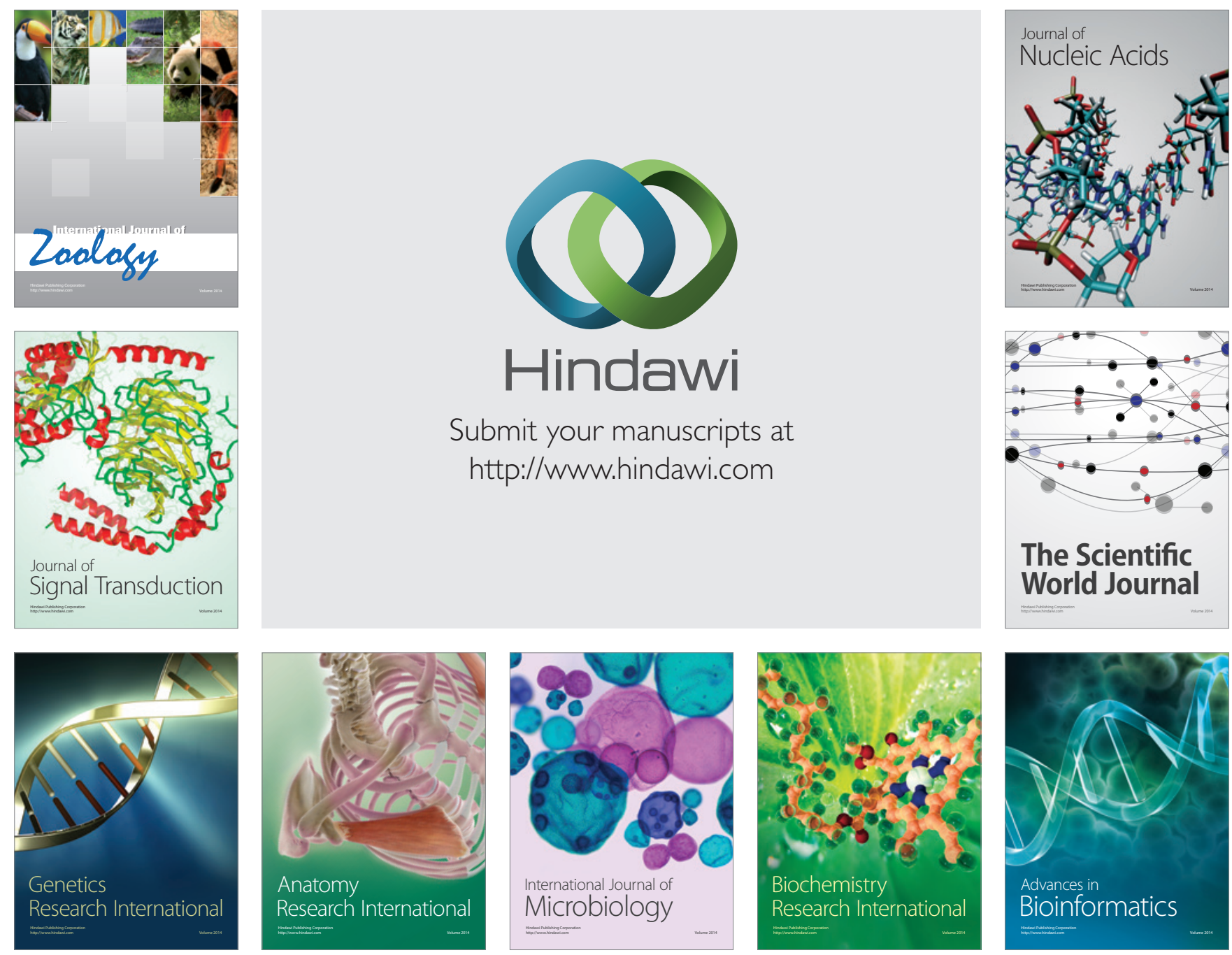

The Scientific World Journal
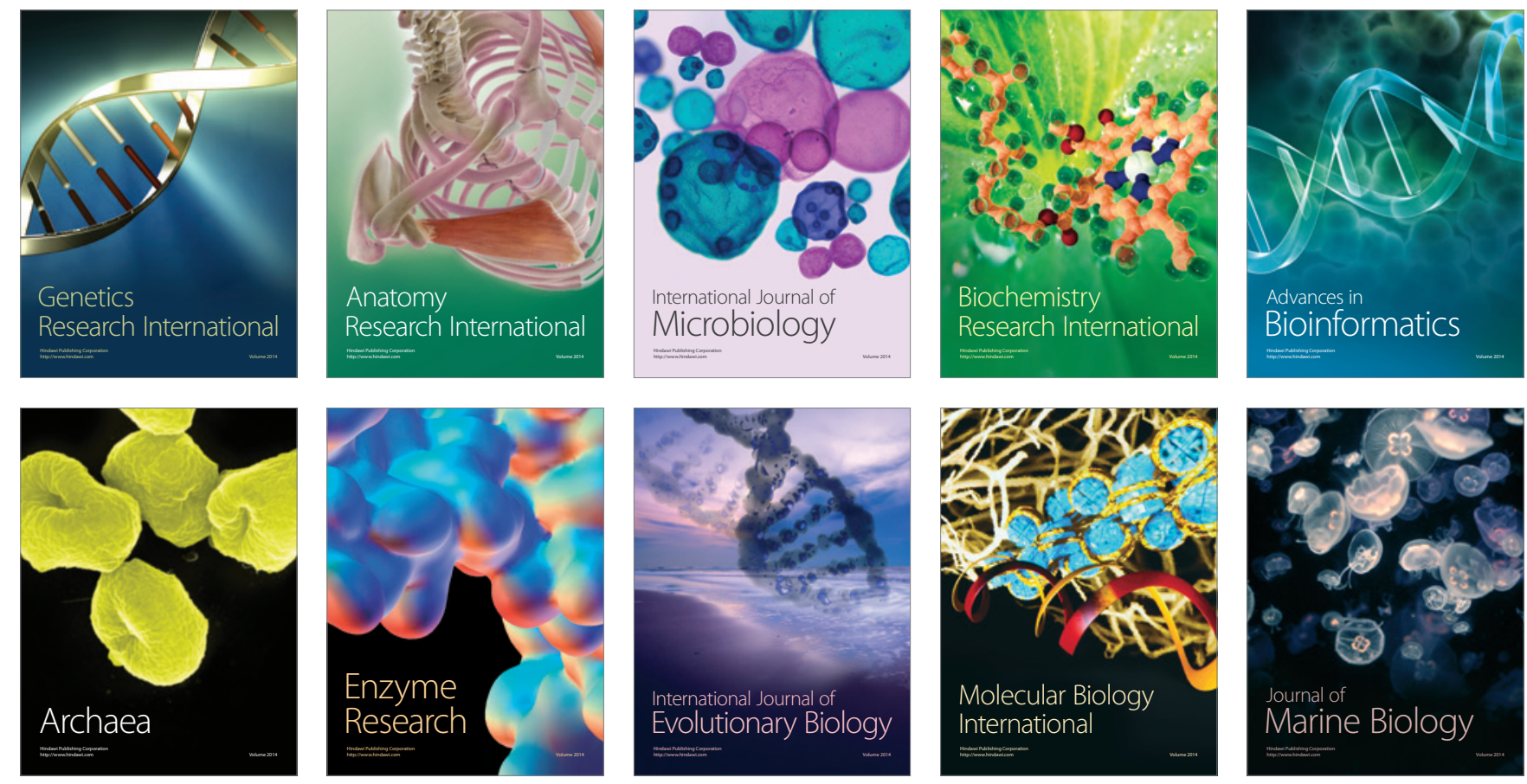